\title{
Alcohol consumption and risk of cardiovascular outcomes and bleeding in patients with established atrial fibrillation
}

\author{
Philipp Reddiess MD, Stefanie Aeschbacher PhD, Pascal Meyre MD PhD, Michael Coslovsky PhD, \\ Michael Kühne MD, Nicolas Rodondi MD MAS, Oliver Baretella MD PhD, Jürg H. Beer MD, Richard Kobza MD, \\ Giorgio Moschovitis MD, Marcello Di Valentino MD, Cyrill Müller MD, Fabienne Steiner MD, Leo H. Bonati MD, \\ Christian Sticherling MD Stefan Osswald MD, David Conen MD MPH; for the BEAT-AF and Swiss-AF \\ investigators*
}

- Cite as: CMAJ 2020 January 25;193:E117-23. doi: 10.1503/cmaj.200778

\begin{abstract}
BACKGROUND: Little is known about the association between alcohol consumption and risk of cardiovascular events in patients with established atrial fibrillation (AF). The main aim of the current study was to investigate the associations of regular alcohol intake with incident stroke or systemic embolism in patients with established AF.
\end{abstract}

METHODS: To assess the association between alcohol consumption and cardiovascular events in patients with established AF, we combined data from 2 comparable prospective cohort studies that followed 3852 patients with AF for a median of 3.0 years. Patients were grouped into 4 categories of daily alcohol intake (none, $>0$ to $<1,1$ to $<2$ and $\geq 2$ drinks/d). The primary outcome was a composite of stroke and systemic embolism. Secondary outcomes were all-cause mortality, myocardial infarction, hospital admission for acute heart failure, and a composite of major and clinically relevant nonmajor bleeding. Associations were assessed using time-updated, multivariable-adjusted Cox proportional hazards models.

RESULTS: Mean age ( \pm standard deviation) was $71 \pm 10$ years ( $28 \%$ were women and $84 \%$ were on oral anticoagulants). We observed 136 confirmed strokes or systemic emboli. Compared with nondrinkers, adjusted hazard ratios for the primary outcome event were $0.87,95 \%$ confidence interval (Cl) 0.55-1.37 for $>0$ to $<1$ drinks/d; $0.70,95 \%$ Cl $0.39-1.25$ for 1 to $<2$ drinks/d; and $0.96,95 \% \mathrm{Cl} 0.56-$ 1.67 for $\geq 2$ drinks/d ( $p$ for linear [quadratic] trend 0.71 [0.22]). There was no significant association between alcohol consumption and bleeding, but there was a nonlinear association with heart failure ( $p$ for quadratic trend 0.01 ) and myocardial infarction ( $p$ for quadratic trend 0.007 ).

INTERPRETATION: In patients with AF, we did not find a significant association between low to moderate alcohol intake and risk of stroke or other cardiovascular events. Our findings do not support special recommendations for patients with established AF with regard to alcohol consumption. Trial registration: ClinicalTrials.gov, no. NCT02105844
A $\mathrm{n}$ association between excessive alcohol intake and atrial arrhythmias was described about 40 years ago as "holiday heart syndrome." ${ }^{\prime \prime}$ since then, several studies have shown that even low amounts of regular alcohol intake are independently associated with an increased risk of incident atrial fibrillation (AF)..$^{2-4}$

In contrast, little is known about the effects of alcohol intake on the risk of adverse events among patients with established AF. ${ }^{5}$ These patients have a high risk of stroke. ${ }^{6,7}$ The use of oral anticoagulation significantly decreases stroke risk, ${ }^{8}$ but a residual risk persists and the risk of bleeding is increased. As alcohol consumption has been associated with stroke risk in non-AF populations, reduced alcohol consumption may help to further mitigate stroke risk in patients with AF. In addition, alcohol consumption may interfere with adherence to oral anticoagulation, increase bleeding risk and lead to more frequent falls, such that the effects of alcohol intake on stroke risk observed in other populations may be not be generalizable to patients with $\mathrm{AF}^{9-14}$

For all these reasons, many physicians recommend to patients with established AF to fully abstain from alcohol. However, these 
recommendations are largely based on expert opinions, and prospective data are needed to address this important gap in knowledge. Therefore, the main aim of our study was to investigate the association of regular alcohol intake with incident stroke or systemic embolism in patients with established AF. As secondary objectives, we also assessed the associations of alcohol intake with incident death, hospital admission for heart failure, myocardial infarction and bleeding.

\section{Methods}

\section{Setting and design}

The Basel Atrial Fibrillation (BEAT-AF) and Swiss Atrial Fibrillation (Swiss-AF) cohort studies are prospective, observational cohort studies conducted in Switzerland. Between 2010 and 2014, BEATAF enrolled 1553 patients with documented AF across 7 centres in Switzerland. BEAT-AF was initiated to assess the feasibility of a multicentre AF cohort study in Switzerland. The main aim of Swiss-AF was to explore the associations of AF with structural and functional brain damage. In Swiss-AF, 2415 patients with documented AF were enrolled between 2014 and 2017 across 14 Swiss centres. Details on the Swiss-AF study design have been published previously. ${ }^{15,16}$ Inclusion criteria for both cohorts were a prior documentation of AF and an age of 65 years or older. Both cohorts enrolled a small convenience sample of patients aged 45-64 years, to assess the effects of AF on individuals in the active workforce. Patients in Beat-AF could not be enrolled in Swiss-AF. In both cohorts, we excluded patients who were unable to sign informed consent, or had only short, potentially reversible AF episodes (e.g., after cardiac surgery or sepsis). Patients with an acute illness within the previous 4 weeks could be enrolled once the acute episode had resolved. To maximize the number of outcome events, we combined both cohorts for this analysis, given the similar eligibility criteria, study setting and study methodology.

From a total of 3968 patients enrolled in both studies, 7 patients were excluded from Beat-AF as they were enrolled in both studies, 67 patients were excluded owing to study termination without having any follow-up information and 42 patients were excluded because of missing information on alcohol intake or covariates, such that 3852 patients remained in this analysis. Baseline characteristics for each cohort are shown in Appendix 1, Table S1, available at www.cmaj.ca/lookup/doi/10.1503/ cmaj.200778/tab-related-content.

\section{Data collection}

In both cohorts, we used identical standardized case report forms to collect information about personal characteristics, risk factors and comorbidities. A copy of the form is provided in Appendix 2, available at www.cmaj.ca/lookup/doi/10.1503/ cmaj.200778/tab-related-content. We calculated body mass index (BMI) as weight in kilograms divided by height in metres squared. We measured blood pressure 3 times in a supine position and used the mean of all values in this analysis. We classified type of AF according to the current European guidelines, as paroxysmal, persistent or permanent. ${ }^{17}$ We collected information on health perception using a visual analogue scale (VAS), scaled from 0 (worst) to 100 (best). During yearly follow-up visits, we updated data on these characteristics, and collected information about adverse outcome events.

\section{Assessment of alcohol consumption}

Using the same standardized case report forms in both cohorts, we asked participants to indicate the average frequency of alcoholic beverages consumed over the last 12 months, and updated alcohol consumption on a yearly basis. One drink was defined as $100 \mathrm{~mL}$ of wine, $20 \mathrm{~mL}$ of liquor, or $300 \mathrm{~mL}$ of beer. For each type of alcoholic beverage, participants had to indicate the frequency of alcohol consumption as never or less than 1 drink per month, 1 to 3 drinks per month, 1 drink per week, 2 to 4 drinks per week, 5 to 6 drinks per week, 1 drink per day, 2 to 3 drinks per day, 4 to 5 drinks per day, or 6 or more drinks per day. To be consistent with previous studies, we categorized the patients into 1 of the following prespecified categories of alcohol intake: ${ }^{3,18}$ nondrinkers, drinkers consuming $>0$ to $<1$ drinks per day, drinkers consuming 1 to $<2$ drinks per day, and drinkers consuming $\geq 2$ drinks per day.

\section{Outcome events}

The primary outcome was a composite of total stroke (ischemic or hemorrhagic) and systemic embolism. Secondary outcomes included a composite of major and clinically relevant nonmajor bleeding, ${ }^{19}$ hospital admission for acute heart failure, myocardial infarction and all-cause mortality. In addition to the information obtained from the patients during the yearly follow-up visits, we also screened all available hospital records for the presence of a possible outcome event. All reported outcome events were independently validated by 2 physicians using standardized definitions. In cases of disagreement, a third physician was consulted. Definitions of these outcomes are provided in Appendix 1, Table S2. Patients and assessors were unaware of the study question.

\section{Statistical analysis}

We stratified baseline characteristics according to alcohol consumption. We calculated person-years of follow-up for all outcomes from the date of the baseline visit to the time of first outcome event of each analysis, dropout, loss to follow-up, death or Apr. 16, 2019.

To compare the associations between categories of alcohol intake and individual outcome events, we constructed timeupdated Cox proportional hazards models to take into account potential changes of alcohol consumption and other covariates over time. We adjusted all regression models for a predefined set of covariates. After adjusting for age and sex in a first step, we adjusted all models for education, hypertension, history of heart failure, history of diabetes, BMI, smoking status, physical activity, history of stroke, history of coronary heart disease, oral anticoagulation, history of renal failure, AF type and health perception. We updated all covariates over time if appropriate. All results are presented as hazard ratios (HRs) and 95\% confidence intervals (Cls) across categories of alcohol intake, using the nondrinkers as 
the reference group. We calculated $p$ values for linear and quadratic trend across categories of alcohol consumption.

We performed several sensitivity analyses. First, to assess the potential influence of the 2 individual cohorts, we additionally adjusted all multivariable models for cohort. Second, to address the possibility that patients with future events may reduce alcohol intake over time because of poor health, we re-ran the main analyses without updating covariates over time. Finally, we performed sex-specific analyses for the associations of alcohol intake with all outcome events. We calculated $p$ values for interaction using multiplicative interaction terms in the nonstratified models. We performed all statistical analyses using $R(R$ version 3.4.1 Vienna, Austria). Estimates are provided with 95\% $\mathrm{Cls}$ and 2-sided $p$ values.

\section{Ethics approval}

The Ethikkommission Nordwest- und Zentralschweiz (EKNZ) approved both study protocols as the lead ethics committee. We obtained informed written consent from all participants.

\section{Results}

Baseline characteristics stratified by alcohol consumption are shown in Table 1. Of the 3852 patients, 689 (18\%) were nondrinkers, $1782(46 \%)$ consumed $>0$ to $<1$ drink per day, $646(17 \%) 1$ to $<2$ drinks per day, and $735(19 \%) \geq 2$ drinks per day. Mean age ( \pm standard deviation) was $71 \pm 10$ years, $28 \%$ were women and $84 \%$ were on anticoagulation at the time of enrolment. It was more likely that patients with higher alcohol intake were male, better educated,

Table 1: Baseline characteristics according to alcohol consumption

\begin{tabular}{|c|c|c|c|c|c|}
\hline Characteristic & $\begin{array}{c}\text { Overall no. }(\%)^{*} \\
\text { of patients } \\
n=3852\end{array}$ & $\begin{array}{c}\text { No. }(\%)^{\star} \\
\text { of patients who } \\
\text { were nondrinkers } \\
n=689\end{array}$ & $\begin{array}{l}\text { No. }(\%)^{\star} \\
\text { of patients who } \\
\text { consumed } \\
>0 \text { to }<1 \text { drink/d } \\
n=1782\end{array}$ & $\begin{array}{l}\text { No. }(\%)^{\star} \\
\text { of patients who } \\
\text { consumed } \\
1 \text { to }<2 \text { drinks/d } \\
n=646\end{array}$ & $\begin{array}{l}\text { No. }(\%)^{\star} \\
\text { of patients who } \\
\text { consumed } \\
\geq 2 \text { drinks/d } \\
n=735\end{array}$ \\
\hline Mean age $\pm S D, y r$ & $71 \pm 10$ & $72 \pm 10$ & $71 \pm 10$ & $73 \pm 10$ & $72 \pm 8$ \\
\hline Female sex & $1078(28)$ & $362(53)$ & $522(29)$ & $114(18)$ & $80(11)$ \\
\hline Ethnicity, White & 3800 (99) & $671(97)$ & $1760(99)$ & 641 (99) & 728 (99) \\
\hline $\mathrm{BMI}, \mathrm{kg} / \mathrm{m}^{2} \pm \mathrm{SD}$ & $27.4 \pm 4.8$ & $28.0 \pm 5.7$ & $27.4 \pm 4.7$ & $27.0 \pm 4.4$ & $27.3 \pm 4.2$ \\
\hline Current smoker & $298(8)$ & $56(8)$ & $117(7)$ & $36(6)$ & $89(12)$ \\
\hline Regular exercise & $1875(49)$ & $265(39)$ & $927(52)$ & $326(51)$ & 357 (49) \\
\hline \multicolumn{6}{|l|}{ Education levelł } \\
\hline Basic & $456(12)$ & $162(24)$ & $171(10)$ & $49(8)$ & $74(10)$ \\
\hline Middle & $1897(49)$ & 369 (54) & $899(50)$ & $311(48)$ & $318(43)$ \\
\hline Advanced & $1499(39)$ & $158(23)$ & $712(40)$ & $286(44)$ & $343(47)$ \\
\hline \multicolumn{6}{|l|}{ Atrial fibrillation type } \\
\hline Paroxysmal & $1899(49)$ & $343(50)$ & $911(51)$ & 319 (49) & $326(44)$ \\
\hline Persistent & $1047(27)$ & $162(24)$ & $493(28)$ & $179(28)$ & $213(29)$ \\
\hline Permanent & $906(24)$ & $184(27)$ & $378(21)$ & $148(23)$ & $196(27)$ \\
\hline AF-related symptoms§ & $2550(66)$ & $468(68)$ & $1237(70)$ & $424(66)$ & $422(57)$ \\
\hline Health perception (VAS)ף & $72 \pm 18$ & $67 \pm 20$ & $73 \pm 18$ & $74 \pm 17$ & $72 \pm 18$ \\
\hline \multicolumn{6}{|l|}{ Medical history } \\
\hline Hypertension & $2645(69)$ & $505(73)$ & $1194(67)$ & $427(66)$ & $519(71)$ \\
\hline Diabetes & $604(16)$ & $153(22)$ & 251 (14) & $87(13)$ & $113(15)$ \\
\hline Stroke or TIA & $663(17)$ & $127(18)$ & 296 (17) & $123(19)$ & $117(16)$ \\
\hline Heart failure & $917(24)$ & $185(27)$ & $420(24)$ & $147(23)$ & $165(22)$ \\
\hline Coronary heart disease & $1031(27)$ & $196(28)$ & $465(26)$ & $166(26)$ & $204(28)$ \\
\hline Renal failure & 716 (19) & $167(24)$ & $316(18)$ & $116(18)$ & $117(16)$ \\
\hline Recurrent falls & $259(7)$ & $66(10)$ & $109(6)$ & $35(5)$ & $49(7)$ \\
\hline Oral anticoagulation & $3248(84)$ & $576(84)$ & $1488(84)$ & $554(86)$ & $630(86)$ \\
\hline \multicolumn{6}{|c|}{$\begin{array}{l}\text { Note: } \mathrm{BMI}=\text { body mass index, } \mathrm{SD}=\text { standard deviation, } \mathrm{TIA}=\text { transient ischemic attack, VAS = visual analogue scale. } \\
\text { *Unless otherwise specified. } \\
\text { †Missing values: } 8 \\
\text { †Basic: } \leq 6 \text { years (less than the current compulsory education curriculum); middle: } 6 \text { to } \leq 12 \text { years (high school or similar); advanced: } \geq 12 \text { years (college or university degree). } \\
\text { SIncludes palpitations, dizziness, chest pain, effort intolerance, dyspnea, fatigue, syncope or other. } \\
\text { IVAS: scale from } 0 \text { (worst) to } 100 \text { (best) health perception. }\end{array}$} \\
\hline
\end{tabular}


active smokers and physically active. They also had a lower prevalence of diabetes, renal failure and AF-related symptoms (Table 1). There was very little change in the distribution of alcohol intake over time, in patients both with and without an incident primary event (Appendix 1, Figure S1). Baseline characteristics stratified by cohort are presented in Appendix 1, Table S1.
After a median follow-up of 3.0 years, the primary outcome of stroke or systemic embolism occurred in 136 patients, with an incidence of 1.01 per 100 person-years of follow-up. The incidence across categories of increasing alcohol intake was 1.29 , $0.90,0.90$ and 1.12 per 100 person-years (Table 2). Compared with nondrinkers, the multivariable adjusted HRs (95\% Cls) for

Table 2: Associations between alcohol consumption and adverse clinical outcome events

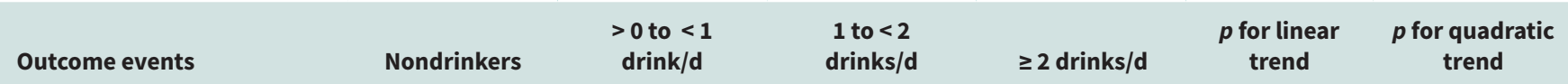

\section{Stroke or systemic embolism}

Events

Person-years

31

Incidence per 100 person-years

2395

1.29

57

6321

0.90

1 (Ref.)

$0.74(0.47-1.16)$

$0.65(0.36-1.14)$

$0.83(0.48-1.43)$

0.43

0.13

Multivariable adjusted*

1 (Ref.)

$0.87(0.55-1.37)$

$0.70(0.39-1.25)$

$0.96(0.56-1.67)$

0.71

0.22

Major bleeding or clinically relevant nonmajor bleeding

\section{Events}

Person-years

Incidence per 100 person-years

Hazard ratio $(95 \% \mathrm{Cl})$

Age and sex adjusted

Multivariable adjusted ${ }^{*}$

115

2240

5.13

1 (Ref.)

1 (Ref.)

Hospital admission for heart failure

Events

Person-years

98

Incidence per 100 person-years

2327

4.21

Hazard ratio $(95 \% \mathrm{Cl})$

Age and sex adjusted

Multivariable adjusted ${ }^{\star}$

1 (Ref.)

$0.75(0.58-0.97)$

$0.41(0.29-0.60)$

$0.75(0.55-1.03)$

0.005

$<0.001$

1 (Ref.)

$1.00(0.77-1.29)$

$0.60(0.41-0.87)$

$1.07(0.78-1.47)$

65

121

2463

4.91

Incidence per 100 person-years

Hazard ratio $(95 \% \mathrm{Cl})$

Age and sex adjusted

Multivariable adjusted ${ }^{\star}$

1 (Ref.)

$0.41(0.32-0.53)$

$0.34(0.24-0.47)$

$0.42(0.31-0.58)$

$<0.001$

$<0.001$

1 (Ref.)

$0.61(0.47-0.79)$

$0.49(0.35-0.69)$

$0.63(0.45-0.86)$

17

2419

0.70

Incidence per 100 person-years

2420

1.45

Hazard ratio $(95 \% \mathrm{Cl})$

Age and sex adjusted

1 (Ref.)

$0.34(0.20-0.55)$

$0.30(0.15-0.59)$

$0.42(0.23-0.78)$

0.007

0.002

1 (Ref.)

$0.41(0.25-0.69)$

$0.39(0.20-0.78)$

$0.55(0.29-1.03)$

0.07

0.007

Note: $\mathrm{Cl}=$ confidence interval, Ref. = reference category.

*Adjusted for age, sex, education, hypertension, history of heart failure, history of diabetes, body mass index, smoking status, physical activity, history of stroke, anticoagulation, history of coronary heart disease, renal failure, atrial fibrillation type and health perception. 
stroke and systemic embolism were 0.87 (0.55-1.37), 0.70 (0.39$1.25)$ and $0.96(0.56-1.67)$ across increasing categories of alcohol consumption. There was no evidence for a linear or nonlinear trend across these categories, as shown in Table 2 and Figure 1. Coefficients for the other model covariates are provided in Appendix 1, Table S3.

Absolute and relative risks for all secondary outcomes are shown in Table 2 and Figure 1. We found no strong evidence for a linear or nonlinear association of alcohol consumption with bleeding. For mortality, there was evidence of a nonlinear association ( $p$ for quadratic trend 0.001), with those drinking 1 to $<2$ alcoholic beverages per day having the lowest risk of death. There was also a nonlinear association between alcohol consumption and myocardial infarction ( $p$ value for quadratic trend 0.007 ), and hospital admission for heart failure ( $p$ for quadratic trend 0.01) (Table 2 and Figure 1). The HRs (95\% Cls) for all covariates are presented in Appendix 1, Table S3.

Additional adjustment for cohort did not change our results (Appendix 1, Table S4). Using baseline covariates only without updating over time resulted in similar findings, as shown in Appendix 1, Table S5. Finally, sex-specific analyses of associations between alcohol consumption and outcome events are shown in Appendix 1, Table S6. In addition, none of the alcohol intake by sex interaction terms were statistically significant.

\section{Interpretation}

In this combined cohort of patients with AF, we found no significant association between alcohol consumption and risk of stroke or systemic embolism. Alcohol consumption was not associated
A) Stroke or systemic embolism

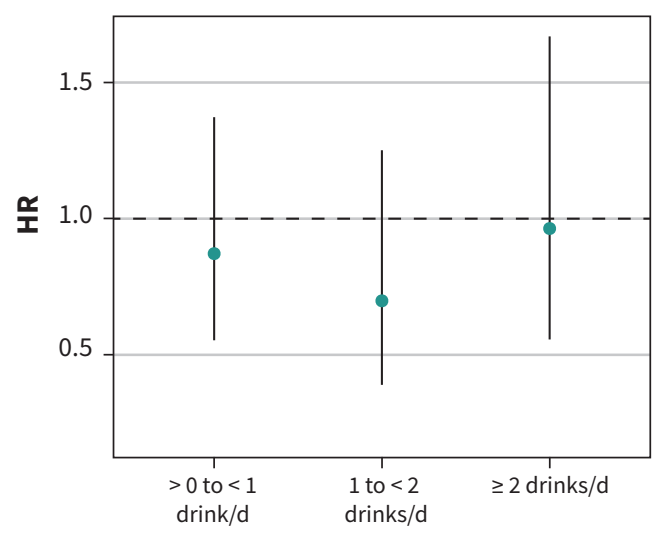

B) Major bleeding or clinically relevant nonmajor bleeding

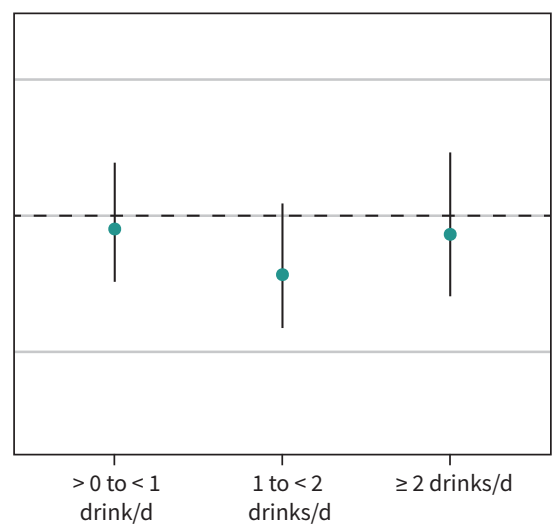

Alcohol consumption

\section{C) Heart failure}

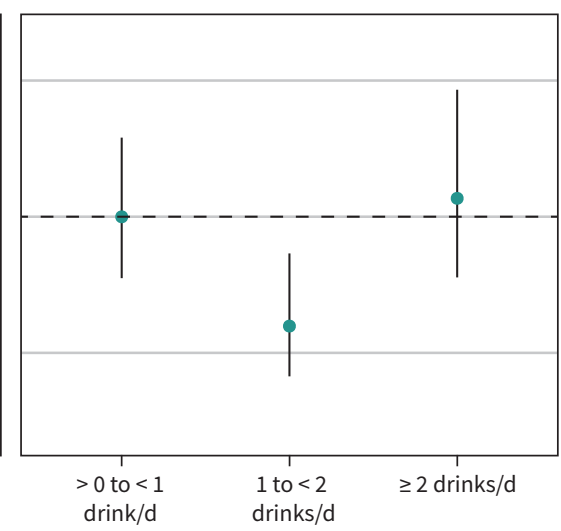

D) Myocardial infarction

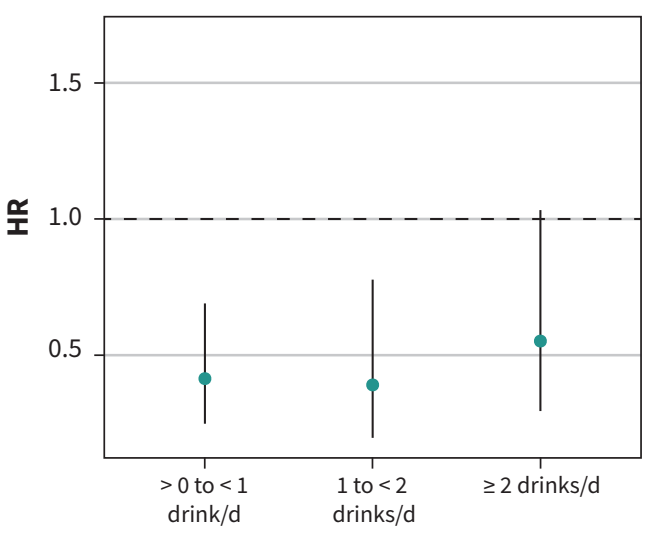

E) All-cause mortality

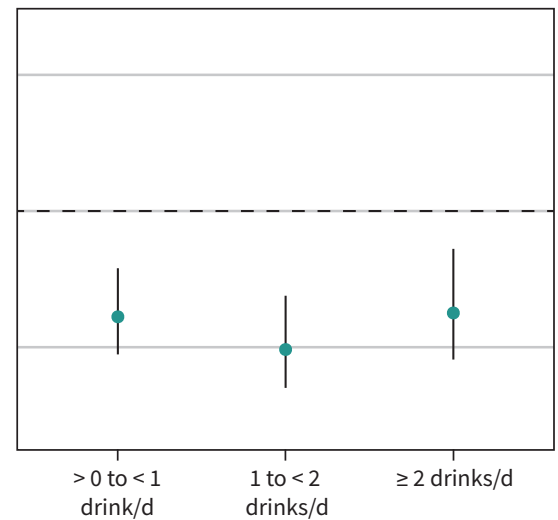

Alcohol consumption

Figure 1: Hazard ratios (HRs) stratified by categories of alcohol consumption: A) stroke or systemic embolism; B) major bleeding or clinically relevant nonmajor bleeding; C) hospital admission for acute heart failure; D) myocardial infarction; E) all-cause mortality. Nondrinkers are the reference group. All risk estimates were adjusted for age, sex, education, hypertension, history of heart failure, history of diabetes, body mass index, smoking status, physical activity, history of stroke or transient ischemic attack, anticoagulation, history of coronary heart disease, renal failure, atrial fibrillation type and health perception. 
with an increased risk of bleeding, despite most participants receiving oral anticoagulation for stroke prevention. We observed nonlinear associations of alcohol intake with mortality, myocardial infarction and hospital admissions for heart failure, similar to associations previously reported in general population samples. ${ }^{20}$

Patients with AF are usually older adults with multiple comorbidities. It is therefore reassuring that in our study, alcohol consumption was not associated with an increased risk of stroke or systemic embolism, although a small effect cannot be excluded given the width of the Cls described in Table 2. Furthermore, the absolute difference in stroke risk between categories of alcohol consumption was small, ranging from 0.9 to 1.27 events per 100 person-years.

Many of these patients need to take oral anticoagulation for stroke prevention and therefore have an increased risk of bleeding. Alcohol consumption may cause further harm by inducing falls, medication errors or medication interactions. It is reassuring that alcohol consumption was not associated with an increased risk of bleeding, with a small difference in absolute risk across drinking categories, although most patients were taking oral anticoagulation.

The associations between alcohol intake and incident cardiovascular events in the general population are well studied.,11,21-23 Many studies have found that low to moderate alcohol consumption was associated with a lower risk of stroke, all-cause mortality or myocardial infarction..$^{23-27}$ In our study of patients with AF, alcohol consumption had similar associations with these outcome events. Our data suggest no excess risk of adverse cardiovascular events among patients with AF who consumed low to moderate amounts of alcohol. Similar recommendations with regard to the safety of alcohol intake as in the general population seem appropriate for patients with AF.

Strengths of this study include the availability of well-characterized patients with AF with an average follow-up of 3 years and with covariates updated on an annual basis. All clinical outcome data were validated. The amount of missing data was very low.

\section{Limitations}

Our study has a number of potential limitations. First, although we controlled for the obvious potential confounders, some unknown residual confounding may persist and could influence the observed associations. Causality cannot be established in an observational study. For instance, nondrinkers tended to have a higher prevalence of several comorbidities (Table 1), raising the possibility of residual confounding by contraindication. Second, alcohol consumption was self-reported. However, studies in other populations have shown that the reliability of selfreported alcohol consumption is usually good. ${ }^{28}$ Nevertheless, the possibility of systematic underreporting of alcohol intake should be considered. Third, virtually all participants in our study were White, and the generalizability of our results to other ethnic groups is unclear. Fourth, the width of the confidence intervals around some of the hazard ratios of association were quite wide. Finally, our study did not include a large number of heavy or binge drinkers, and our results may therefore not apply to these patients.

\section{Conclusion}

In this study of patients with AF, low to moderate alcohol intake was not associated with an increased risk of stroke and other cardiovascular events. Alcohol intake was not associated with an increased risk of bleeding. The observed associations were similar to those in previous general population studies. Therefore, our findings do not support special recommendations for patients with AF with respect to alcohol consumption.

\section{References}

1. Ettinger PO, Wu CF, De La Cruz C Jr, et al. Arrhythmias and the "Holiday Heart": alcohol-associated cardiac rhythm disorders. Am Heart J 1978;95:555-62.

2. Djoussé L, Levy D, Benjamin EJ, et al. Long-term alcohol consumption and the risk of atrial fibrillation in the Framingham Study. Am J Cardiol 2004;93:710-3.

3. Conen D, Tedrow UB, Cook NR, et al. Alcohol consumption and risk of incident atrial fibrillation in women. JAMA 2008;300:2489-96.

4. Larsson SC, Drca N, Wolk A. Alcohol consumption and risk of atrial fibrillation: a prospective study and dose-response meta-analysis. J Am Coll Cardiol 2014; 64:281-9.

5. Kwon Y, Norby FL, Jensen PN, et al. Association of smoking, alcohol, and obesity with cardiovascular death and ischemic stroke in atrial fibrillation: the Atherosclerosis Risk in Communities (ARIC) study and Cardiovascular Health Study (CHS). PLoS One 2016;11:e0147065.

6. Healey JS, Hart RG, Pogue J, et al. Risks and benefits of oral anticoagulation compared with clopidogrel plus aspirin in patients with atrial fibrillation according to stroke risk: the atrial fibrillation clopidogrel trial with irbesartan for prevention of vascular events (ACTIVE-W). Stroke 2008;39:1482-6.

7. Conen D, Chae CU, Glynn RJ, et al. Risk of death and cardiovascular events in initially healthy women with new-onset atrial fibrillation. JAMA 2011;305: 2080-7.

8. Hart RG, Pearce LA, Aguilar MI. Meta-analysis: antithrombotic therapy to prevent stroke in patients who have nonvalvular atrial fibrillation. Ann Intern Med 2007;146:857-67.

9. Mostofsky E, Chahal HS, Mukamal KJ, et al. Alcohol and immediate risk of cardiovascular events: a systematic review and dose-response meta-analysis. Circulation 2016;133:979-87.

10. Conen D. Alcohol consumption and incident cardiovascular disease: not just one unifying hypothesis. Eur Heart J 2015;36:897-8.

11. Reynolds K, Lewis B, Nolen JD, et al. Alcohol consumption and risk of stroke: a meta-analysis [published erratum in JAMA 2003;289:2798]. JAMA 2003;289:579-88.

12. Holton AE, Gallagher PJ, Ryan C, et al. Consensus validation of the POSAMINO (POtentially Serious Alcohol-Medication INteractions in Older adults) criteria. BMJ Open 2017;7:e017453.

13. Weathermon R, Crabb DW. Alcohol and medication interactions. Alcohol Res Health 1999;23:40-54.

14. Mukamal KJ, Mittleman MA, Longstreth WT Jr, et al. Self-reported alcohol consumption and falls in older adults: cross-sectional and longitudinal analyses of the cardiovascular health study. J Am Geriatr Soc 2004;52:1174-9.

15. Conen D, Rodondi N, Mueller A, et al. Design of the Swiss Atrial Fibrillation Cohort Study (Swiss-AF): structural brain damage and cognitive decline among patients with atrial fibrillation. Swiss Med Wkly 2017;147:w14467.

16. Conen D, Rodondi N, Muller A, et al. Relationships of Overt and Silent Brain Lesions With Cognitive Function in Patients With Atrial Fibrillation. J Am Coll Cardiol 2019;73:989-99.

17. Camm AJ, Kirchhof P, Lip GY, et al; ESC Committee for Practice Guidelines. Guidelines for the management of atrial fibrillation: the Task Force for the Management of Atrial Fibrillation of the European Society of Cardiology (ESC). Europace 2010;12:1360-420.

18. Hvidtfeldt UA, Tolstrup JS, Jakobsen MU, et al. Alcohol intake and risk of coronary heart disease in younger, middle-aged, and older adults. Circulation 2010;121:1589-97.

19. Schulman S, Kearon C. Definition of major bleeding in clinical investigations of antihemostatic medicinal products in non-surgical patients. J Thromb Haemost 2005;3:692-4. 
20. Larsson SC, Wallin A, Wolk A. Contrasting association between alcohol consumption and risk of myocardial infarction and heart failure: two prospective cohorts. Int J Cardiol 2017;231:207-10.

21. Stampfer MJ, Colditz GA, Willett WC, et al. A prospective study of moderate alcohol consumption and the risk of coronary disease and stroke in women. $N$ Engl J Med 1988;319:267-73.

22. Mukamal K, Lazo M. Alcohol and cardiovascular disease. BMJ 2017;356:j1340.

23. Chiuve SE, Rimm EB, Mukamal KJ, et al. Light-to-moderate alcohol consumption and risk of sudden cardiac death in women. Heart Rhythm 2010;7:1374-80.

24. Bell S, Daskalopoulou M, Rapsomaniki E, et al. Association between clinically recorded alcohol consumption and initial presentation of 12 cardiovascular diseases: population-based cohort study using linked health records. BMJ 2017;356:j909.
25. Berger K, Ajani UA, Kase CS, et al. Light-to-moderate alcohol consumption and the risk of stroke among U.S. male physicians. N Engl J Med 1999;341:1557-64.

26. Ricci C, Wood A, Muller D, et al. Alcohol intake in relation to non-fatal and fatal coronary heart disease and stroke: EPIC-CVD case-cohort study. BMJ 2018; 361:k934.

27. Yusuf S, Hawken S, Ounpuu S, et al. Effect of potentially modifiable risk factors associated with myocardial infarction in 52 countries (the INTERHEART study): case-control study. Lancet 2004;364:937-52.

28. Giovannucci E, Colditz G, Stampfer MJ, et al. The assessment of alcohol consumption by a simple self-administered questionnaire. Am J Epidemiol 1991;133:810-7.
*See Appendix 1, available at www.cmaj.ca/lookup/doi/10.1503/ cmaj.200778/tab-related-content for a list of all investigators.

Competing interests: Jürg Beer reports receiving grants from the Swiss National Foundation of Science and the Swiss Heart Foundation. Richard Kobza reports receiving institutional grants from Abbott, Biosense-Webster, Boston-Scientific, Biotronik, Medtronic and SisMedical. Giorgio Moschovitis reports receiving advisory board fees from Boehringer Ingelheim, AstraZeneca and Novartis, outside of the submitted work. Michael Kühne reports receiving personal fees from Bayer, Böhringer Ingelheim, Pfizer BMS, Daiichi Sankyo, Medtronic, Biotronik, Boston Scientific and Johnson \&Johnson, and grants from Bayer, Pfizer BMS, Boston Scientific, the Swiss National Science Foundation and the Swiss Heart Foundation. Leo Bonati reports receiving grants from the Swiss National Science Foundation, the Swiss Heart Foundation and the University of Basel; grants and nonfinancial support from Bayer and AstraZeneca; personal fees from Amgen, Bristol-Myers Squibb and Claret Medical, outside the submitted work. Stefan Osswald reports receiving research grants from the Swiss National Science Foundation, the Swiss Heart Foundation and the Cardiovascular Research Foundation, Basel. David Conen reports receiving speaker fees from Servier, Canada, outside of the current work. No other competing interests were declared.

This article has been peer reviewed.

Affiliations: Cardiovascular Research Institute Basel (Reddiess, Aeschbacher, Meyre, Coslovsky, Kühne, Müller, Steiner, Sticherling, Osswald, Conen); Cardiology Division (Reddiess, Aeschbacher, Meyre, Coslovsky, Kühne, Müller, Steiner, Sticherling, Osswald); Clinical Trial Unit Basel, Department of Clinical Research (Coslovsky), University Hospital Basel, Switzerland; Institute of Primary Health Care (BIHAM) (Rodondi), University of Bern; Department of General Internal Medicine (Rodondi), Inselspital, Bern University Hospital, University of Bern, Bern, Switzerland; Department of Medicine (Beer), Cantonal Hospital of Baden and Molecular Cardiology, University Hospital of Zürich, Switzerland; Department of Cardiology (Kobza), Luzerner Kantonsspital, Switzerland; Department of Cardiology (Moschovitis), EOC Ospedale Regionale di Lugano, Switzerland; Department of Cardiology (Di Valentino), EOC Ospedale San Giovanni, Bellinzona, Switzerland; Department of Neurology and Stroke Center (Bonati),
University Hospital Basel, University of Basel, Switzerland; Population Health Research Institute (Conen), McMaster University, Hamilton, Ont.

Contributors: Philipp Reddiess and Stefanie Aeschbacher contributed equally, as shared first authors.Philipp Reddiess, Stefanie Aeschbacher, Stefan Osswald and David Conen conceived and designed the study. Philipp Reddiess, Stefanie Aeschbacher, Pascal Meyre, Michael Kühne, Nicolas Rodondi, Oliver Baretella, Jürg Beer, Richard Kobza, Giorgio Moschovitis, Marcello Di Valentino, Cyrill Müller, Fabienne Steiner, Leo Bonati, Christian Sticherling, Stefan Osswald and David Conen acquired the data. Philipp Reddiess, Stefanie Aeschbacher and Michael Coslovsky analyzed the data. All authors interpreted the data. Philipp Reddiess, Stefanie Aeschbacher and David Conen wrote the first draft. All authors critically revised the manuscript for important intellectual content. All authors approved the final version.

Funding: The Beat-AF study was supported by the Swiss National Science Foundation (Grant number PP00P3_159322), the Swiss Heart Foundation, the University of Basel, Boehringer Ingelheim, SanofiAventis, Merck Sharp \& Dome, Bayer, Daiichi-Sankyo and Pfizer/BristolMyers Squibb. The Swiss-AF cohort study is supported by grants from the Swiss National Science Foundation (grant numbers 33CS30_148474 and 33CS30_177520), the Foundation for Cardiovascular Research Basel and the University of Basel. David Conen holds a McMaster University Department of Medicine Mid-Career Research Award. None of the funders had any role in manuscript preparation or in the decision to submit the manuscript for publication.

Content licence: This is an Open Access article distributed in accordance with the terms of the Creative Commons Attribution (CC BY-NC-ND 4.0) license, which permits use, distribution and reproduction in any medium, provided that the original publication is properly cited, the use is noncommercial (i.e. research or educational use), and no modifications or adaptations are made. See: https://creativecommons.org/licenses/ by-nc-nd/4.0/

Data sharing: Data related to this work are not available for use by other researchers.

Accepted: Sept. 1, 2020

Correspondence to: David Conen, Conend@mcmaster.ca 